\title{
Nucleosynthesis in Binary Populations
}

\author{
Robert G. Izzard and Christopher A. Tout \\ Institute of Astronomy, Madingley Road, Cambridge CB3 0HA, UK \\ rgi@ ast.cam.ac.uk \\ Received 2003 May 2, accepted 2003 June 16
}

\begin{abstract}
We investigate the effect of duplicity on stellar yields of carbon, nitrogen, and oxygen. Populations of single and binary stars are modelled and the yields calculated for the whole population. The effects of explosive nucleosynthesis in novae and supernovae are included but by artificially removing these effects from our populations we determine the influence of a binary companion on asymptotic giant branch yields of the CNO elements.
\end{abstract}

Keywords: stars: AGB and post-AGB — binaries: general — stars: abundances

\section{Introduction}

For many years the study of stellar yields and galactic chemical evolution (GCE) has gone on assuming, mainly for simplicity, that stars are isolated objects (one exception being De Donder \& Vanbeveren 2002). Reality bites deeply into this picture with the observation that most stars are in multiple systems and that many of these systems are interacting. The state of the art in binary star nucleosynthesis is focused on explosive events such as type Ia supernovae and classical novae but other binary star processes contribute to pollution of the interstellar medium. Mass transfer by Roche-lobe overflow (RLOF) occurs particularly when the stellar radius is growing rapidly and so commonly when one star is on the asymptotic giant branch (AGB). Here we evolve populations of single and binary stars using a rapid evolution code and compare the yields of CNO.

\section{Modelling Nucleosynthesis}

We have extended our rapid binary star evolution (BSE; Hurley, Tout, \& Pols 2002) code to include nucleosynthesis in low and intermediate mass stars. The basis of the BSE model is the single star evolution (SSE; Hurley Pols, \& Tout 2000) model with nucleosynthesis which we describe first, followed by the binary aspects of the code.

We have added a synthetic nucleosynthesis package (Izzard et al. 2003) to run in parallel with the original SSE code. The models currently deal with hydrogen, helium, ${ }^{12} \mathrm{C},{ }^{13} \mathrm{C},{ }^{14} \mathrm{~N}$, and ${ }^{16} \mathrm{O}$. First dredge-up is fitted to the detailed models (Pols et al. 1998) on which the SSE code was based. Second dredge-up, the minimum mass for third dredge-up, third dredge-up efficiency and hotbottom burning of the CNO isotopes in thermally pulsing AGB (TPAGB) stars are included as functions of mass and metallicity based on the non-overshooting models of the Monash group (Karakas, Lattanzio, \& Pols 2002). Wind mass loss is included according to the prescription of Hurley et al. (2002) prior to the AGB and Karakas et al. (2002) when the star is on the AGB. The mass loss rate $\dot{M}$ is then given by the formula of Vassiliadis \& Wood (1993)

$$
\log _{10}\left(\dot{M} / \mathrm{M}_{\odot} \mathrm{yr}^{-1}\right)=-11.4+0.0125(P / \mathrm{d}),
$$

where $P$ is the Mira pulsation period given by

$$
\begin{aligned}
\log _{10}(P / \mathrm{d})= & -2.07-0.9 \log _{10}\left(M / \mathrm{M}_{\odot}\right) \\
& +1.94 \log _{10}\left(R / \mathrm{R}_{\odot}\right)
\end{aligned}
$$

with $M$ the mass of the star and $R$ its radius.

On the TPAGB and for $P \geq 500 \mathrm{~d}$ the rate in equation (1) is truncated where necessary to a maximal superwind of rate

$$
\dot{M}=\frac{L}{c v_{\exp }},
$$

where $c$ is the speed of light and the expansion velocity of the wind,

$$
v_{\exp }=\min [(-13.5+0.056 P / \mathrm{d}), 15] \mathrm{km} \mathrm{s}^{-1} .
$$

For the range of masses considered here $\left(0.1 \leq M / \mathrm{M}_{\odot} \leq\right.$ 8.0 , at solar metallicity) supernovae do not occur in our single star models.

In adding nucleosynthesis to the BSE code we have dealt with the following scenarios.

- Core-collapse supernovae (CC-SNe, both Type II SNe (SNeII) and Type Ib/c SNe (SNeIb/c)) are added with the yields of Woosley \& Weaver (1995) with a correction for envelope mass loss according to Portinari, Chiosi, \& Bressan (1998). Merging binaries are the main source of CC-SNe in the stars considered here.

- Type Ia supernovae (SNeIa) are thought to be the result of mass transfer in double white dwarf binaries. We include accreting or merging helium white dwarfs Woosley, Taam, \& Weaver (1986), edge-lit detonations of carbon-oxygen white dwarfs (COWDs) in sub$M_{\mathrm{Ch}}$ explosions from Livne \& Arnett (1995) and near $M_{\mathrm{Ch}}$ explosions of accreting or merging COWDs with 
the DD2 model of Iwamoto et al. (1999). Accretioninduced collapse SNe (Nomoto \& Kondo 1991) are treated as having zero yield.

- Novae are included by fitting ejecta abundances to the models of José \& Hernanz (1998) for CO and ONeMg white dwarfs.

- RLOF is treated by the BSE model and we use this prescription unchanged but keep track of the composition of the overflowing matter. As well as SNeIa and novae the RLOF process can affect binary evolution by truncation of phases of evolution when the stellar radius is large, such as on the giant branch and AGB. Non-conservative RLOF leads to a direct contribution to the stellar yield. Conservative mass transfer pollutes the companion star (see below).

- In some cases a common envelope (CE) forms around both stars. Part of this envelope may be driven off and contribute directly to the stellar yield. The end of the CE phase depends on whether the cores of the progenitor stars merge. If this does happen it is possible for a new TPAGB star to form. Due to the general lack of understanding of the CE we do not yet include any additional nucleosynthesis such as that described by Ivanova, Podsiadlowski, \& Spruit (2002).

- Accretion of mass from a companion can occur during RLOF or from a companion's stellar wind. The BSE model contains a standard Bondi-Hoyle type (Bondi \& Hoyle 1944) wind accretion which we use unchanged from Hurley et al. (2002) but with care over the composition of the accreting material. If one star (the donor) has a significant wind but the other (the accretor) does not there is no problem and all the accreting material is from the donor. However if both stars have a significant wind it is unlikely that all the accreting material is from the donor; some will be from the accretor. To deal with this we consider the momentum flux from each stellar wind and find the point in space where these fluxes are equal in magnitude (Huang \& Weigert 1982). If this point is beyond the accreting star then the accreted material is all from the donor; if it is between the stars then a shock is assumed to form such that no material from the donor reaches the accretor and excess material flows out of the system to enrich the ISM. This simple model ignores rotation (stellar or orbital), accretion discs and all sources of extra mixing. For the RLOF case the material falls directly onto the companion's surface.

- The surface abundance of the accreting star is affected by accretion. We use a simple two-layer model consisting of the stellar envelope and an accretion layer. We compare the molecular weight $\mu$ of the two layers and if $\mu_{\text {acc }}>\mu_{\text {env }}$ we mix the layers instantaneously by a thermohaline instability. Otherwise the accreted layer remains on the surface. The most common case of accretion is onto a main-sequence star from an evolved companion and in this case the evolved stellar material is denser than that of the main-sequence star so it always sinks and mixes and the effect on surface abundance is diluted.

\section{Population Synthesis}

We evolved a population of single stars and of binary stars at solar metallicity from the zero-age main sequence to an age of $13.7 \mathrm{Gyr}$, the maximum possible age of the Galaxy (Bennett et al. 2003). We use the initial mass function (IMF) for single stars of Kroupa, Tout, \& Gilmore (1993, KTG93) with $0.1 \leq M / \mathrm{M}_{\odot} \leq 8.0$. For binaries we choose the primary (mass $M_{1}$ ) from the KTG93 IMF with the secondary (mass $M_{2}$ ) according to a flat distribution in $q=M_{2} / M_{1}$ for $q \leq 1$. The separation $a$ is taken to be a flat distribution in $\log a$ with $3 \leq a / \mathrm{R}_{\odot} \leq 10^{4}$. When $a>10^{4} \mathrm{R}_{\odot}$ there is no binary interaction so both components enrich as single stars. The results of Hurley et al. (2002) indicate that a distribution of non-zero eccentricities has little effect if $a$ are taken to be the semi-latera recta $l$ of the binary orbits because orbital angular momentum depends only on $l$ and any eccentric systems tidally circularise before interacting. Each binary population consists of $N^{3}$ stars on a logarithmic grid, with $N=100$. The single star population is on a grid of $10 \mathrm{~N}$ stars logarithmically spaced in $M$. Note that while our choice of mass limits includes most stars it does not include Wolf-Rayet (WR) stars because $\max \left(M_{1}+M_{2}\right)=16 \mathrm{M}_{\odot}$ in a merged binary if $M_{1} \leq 8 \mathrm{M}_{\odot}$ and $M_{2} \leq 8 \mathrm{M}_{\odot}$. The yields of WR stars are important but modelling them is a complex process that we leave to future work. Here we concentrate on AGB stars.

We use a simple definition of the yield, the mass of an isotope ejected into space from the system integrated over the population,

$$
y_{i}=\int_{0.1 \mathrm{M}_{\odot}}^{8 \mathrm{M}_{\odot}} \int_{0.1}^{M_{1}} \int_{\mathrm{M}_{\odot}}^{10^{4} \mathrm{R}_{\odot}} \int_{0}^{t} \dot{M} X_{i} \psi\left(M_{1}, M_{2}, a\right) d t^{\prime} d a d M_{2} d M_{1}
$$

where $\dot{M}$ is the rate of mass lost from the system, $X_{i}$ is the mass fraction of isotope $i$ in the ejecta, $\psi\left(M_{1}, M_{2}, a\right)$ is the appropriate distribution function (described above), and $t=13.7$ Gyr. However the use of $y_{i}$ to compare single and binary stars is biased against the single stars because more mass is put into a binary star system (see Table 1 for a comparison). We can look at either the yield relative to the mass put into stars or the mass fraction of a given isotope in the ejecta.

Though it is beyond the scope of this paper to explore the large binary parameter space and associated variations of yields by changing the initial distributions or free parameters and this will be addressed in future work, we do not expect qualitative differences. Resolution is not an

Table 1. Mass input and output from each population of stars for the distributions given in Section 3. Binary stars are marginally more efficient at ejecting mass than their solitary counterparts in the case of mass distribution we have used

\begin{tabular}{lccc}
\hline & Binary & Single & Bin/Sin Ratio \\
\hline Mass into stars $/ \mathrm{M}_{\odot}$ & 0.739 & 0.458 & 1.61 \\
Mass ejected to ISM $/ \mathrm{M}_{\odot}$ & 0.191 & 0.115 & 1.66 \\
\hline
\end{tabular}


issue because the results converge on a stable solution for $N$ greater than about 40 so setting $N=100$ is quite safe.

\section{Results}

Table 2 shows the ratio of binary to single star yields for the populations described above, relative to mass both input and ejected. For GCE models the left column, relative to mass input, is probably more useful (most GCE codes use this) although the values are similar for both. Hydrogen, helium and ${ }^{12} \mathrm{C}$ show little change while there is overproduction of ${ }^{13} \mathrm{C}$ and ${ }^{16} \mathrm{O}$ and an underproduction of ${ }^{14} \mathrm{~N}$. The overproduction is due to explosive nucleosynthesis. Our novae models eject substantial amounts of ${ }^{13} \mathrm{C}$ but only a little ${ }^{14} \mathrm{~N}$ while supernovae are responsible for the ${ }^{16} \mathrm{O}$, some ${ }^{12} \mathrm{C}$ but not ${ }^{14} \mathrm{~N}$. Hydrogen and helium are almost the same because these are ejected from unprocessed layers forming the bulk of the stars. If we exclude the explosive yields (Table 3 ) we can see that the $\mathrm{CN}$ isotopes are reduced in binaries. The reason for this lies in the other major source of CNO, the AGB stars. Binary interaction can truncate the AGB before significant third dredge-up and hot-bottom burning begin so $\mathrm{C}$ and ${ }^{14} \mathrm{~N}$ production drops. While ${ }^{16} \mathrm{O}$ is both dredged-up and burned both effects are so small that the ratio is 1.0. The reduction in the ${ }^{12} \mathrm{C}$ yield relative to single stars and normalised to the mass put into stars is $14 \%$, for ${ }^{13} \mathrm{C} 37 \%$ and for ${ }^{14} \mathrm{~N}$ $31 \%$. Comparison of Tables 2 and 3 shows this effect is cancelled out by explosive nucleosynthesis in the case of ${ }^{12} \mathrm{C}$ while ${ }^{13} \mathrm{C}$ and ${ }^{16} \mathrm{O}$ are overproduced by novae and $\mathrm{SNe}$ respectively. Nitrogen is depleted by $26 \%$ even when novae and supernovae are included because its only major source is the AGB stars.

To further investigate the contribution from various sources we compare the yield from a single star of a given mass with a population of binaries with the same primary mass as the single star. For single stars the weight assigned to each star is just given by the IMF but for binaries we must integrate over the secondary and separation distributions such that the yield calculated is for a slice of the $3 \mathrm{D}$ parameter space with the same probability as a single star. ${ }^{1}$ We consider the mass range $0.5 \leq M_{1} / \mathrm{M}_{\odot} \leq 8.0$ for the primary and $0.1 \leq M_{2} / \mathrm{M}_{\odot} \leq M_{1}$ for the secondary because there is no evolution within the age of the Galaxy if $M_{1} \leq 0.5$ while even a $0.1 \mathrm{M}_{\odot}$ companion can truncate the AGB of a more massive primary. It is not so much the cutoff at $0.1 \mathrm{M}_{\odot}$ that matters but the total fraction of such low-mass companions.

Figure 1 shows the yields of the $\mathrm{CNO}$ isotopes relative to the initial mass input to stars. The suppression of third dredge-up by RLOF and hence destruction of AGB stars can be seen for ${ }^{12} \mathrm{C}$ in the mass range $2.5<M / \mathrm{M}_{\odot}<5.0$ while the effect of $\mathrm{SNe}$ dominates as the mass of the binary increases. The plot for ${ }^{13} \mathrm{C}$ shows the narrow peak expected for single stars (see Izzard et al. 2003 for a full

\footnotetext{
${ }^{1}$ This is equivalent to integrating equation (5) for $t^{\prime}, a$, and $M_{2}$ but not $M_{1}$, although the IMF factor is still included.
}

Table 2. Ratio of binary to single star yield for $\mathbf{H}$, He, and CNO isotopes. The left column is for yields normalised to mass input to stars, the right column is for the mass fraction in the ejecta

\begin{tabular}{lcc}
\hline & $\begin{array}{c}\text { Bin/Sin relative } \\
\text { to mass input }\end{array}$ & $\begin{array}{c}\text { Bin/Sin relative mass } \\
\text { fraction in ejecta }\end{array}$ \\
\hline${ }^{1} \mathrm{H}$ & 1.0 & 1.0 \\
${ }^{4} \mathrm{He}$ & 0.98 & 0.95 \\
${ }^{12} \mathrm{C}$ & 1.0 & 1.0 \\
${ }^{13} \mathrm{C}$ & 1.7 & 1.6 \\
${ }^{14} \mathrm{~N}$ & 0.74 & 0.72 \\
${ }^{16} \mathrm{O}$ & 1.4 & 1.3 \\
\hline
\end{tabular}

Table 3. As Table 2 without novae and supernovae. Notice that the way we calculate the yields is now irrelevant

\begin{tabular}{lcc}
\hline & $\begin{array}{c}\text { Bin/Sin relative } \\
\text { to mass input }\end{array}$ & $\begin{array}{c}\text { Bin/Sin relative mass } \\
\text { fraction in ejecta }\end{array}$ \\
\hline${ }^{1} \mathrm{H}$ & 1.0 & 1.0 \\
${ }^{4} \mathrm{He}$ & 0.96 & 0.96 \\
${ }^{12} \mathrm{C}$ & 0.86 & 0.86 \\
${ }^{13} \mathrm{C}$ & 0.63 & 0.63 \\
${ }^{14} \mathrm{~N}$ & 0.69 & 0.69 \\
${ }^{16} \mathrm{O}$ & 1.0 & 1.0 \\
\hline
\end{tabular}

discussion and comparison of single star yields to previous results) and the larger and broader peak from novae. Nitrogen is produced about equally in single and binary stars with $M \lesssim 5 \mathrm{M}_{\odot}$ because there is no hot-bottom burning but above this mass the truncation of the AGB phase in binaries limits nitrogen production and single stars are dominant by a factor of two. Oxygen production is dominated by SNeII as $M$ increases but there is some enhancement at low mass owing to novae and SNeIa.

Again we look at the same data without explosive nucleosynthesis (Figure 2) to focus on the AGB stars. Single stars dominate the ${ }^{12} \mathrm{C}$ yield because third dredge-up is suppressed in binaries. The yield of ${ }^{13} \mathrm{C}$ is much greater for single stars, with very little partial $\mathrm{CN}$ processing in AGB stars in binaries, as expected. The nitrogen yield is very similar to the case with explosive nucleosynthesis included. Oxygen yields for single and binary stars are very similar once supernovae are removed.

It is also interesting to cut slices through the $M_{1}-M_{2}-P$ binary parameter space to identify regions where isotopic production is enhanced or suppressed. Figure 3 shows the binary/single nitrogen yield ratio in the secondary massinitial period plane for a primary mass of $M_{1}=7 \mathrm{M}_{\odot}$. Yields are relative to mass input to stars. Ratios are in the range 0 to 1 only. Dark colours (blue/black) represent the most severe lack of nitrogen, while light colours (yellow/white) indicate a yield similar to the single stars. The evolution of some systems is given in Figure 4, which can be overlaid on Figure 3. Note that binary evolution is a complicated business: some stars have multiple common 

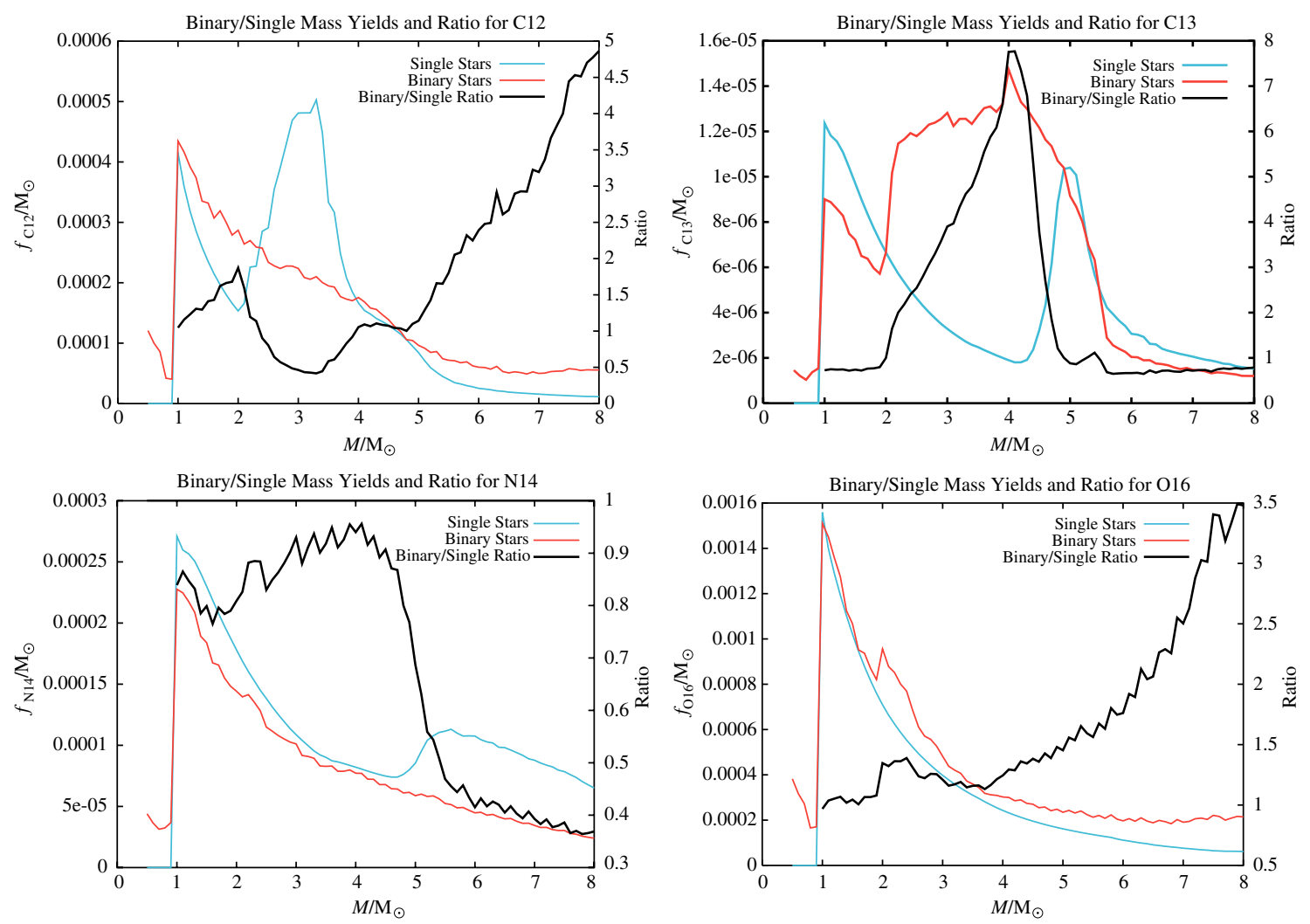

Figure 1 CNO yields (relative to the initial mass put into stars) for single and binary stars with the same primary mass. The left axis is for the yields and the right axis is the binary/single yield ratio. The quantity $f_{i} d M$ is the mass of species $i$ returned to the ISM by single stars of mass between $M$ and $M+d M$ or binary stars with primary mass between $M$ and $M+d M$ for every $1 \mathrm{M}_{\odot}$ formed into stars with masses or primary masses between 0.1 and $8 \mathrm{M}_{\odot}$.
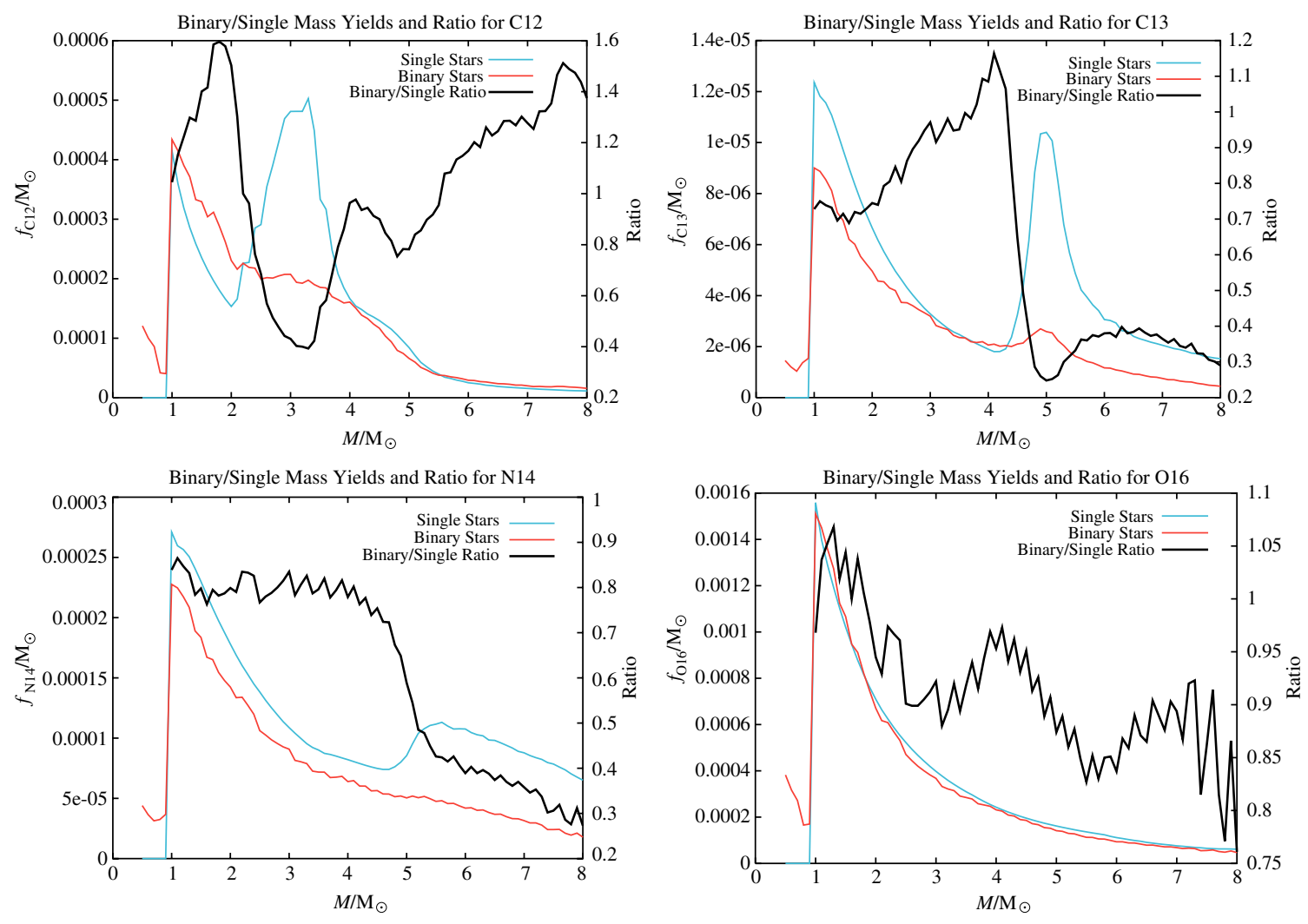

Figure 2 As Figure 1 without supernovae and novae. 


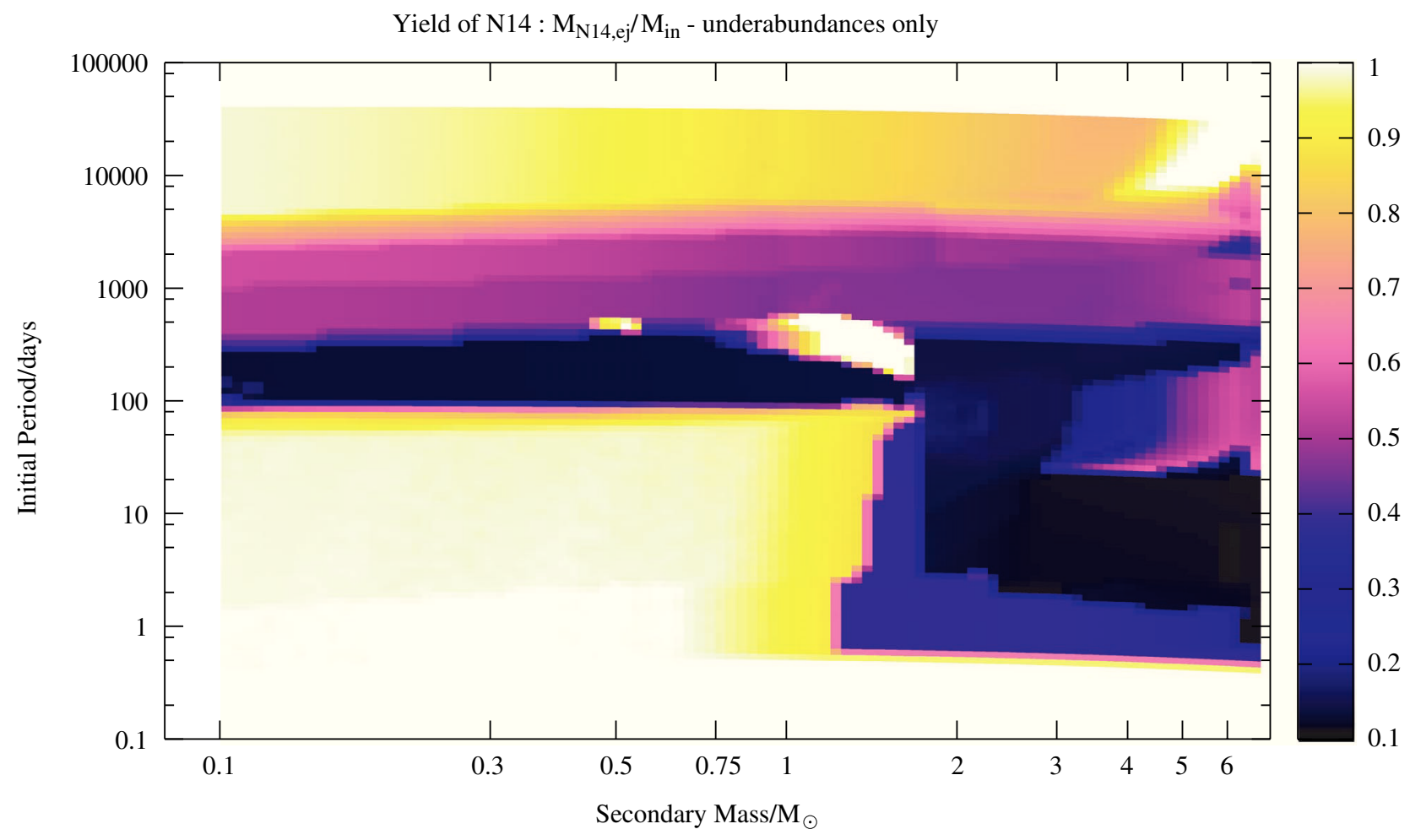

Figure $3{ }^{14} \mathrm{~N}$ binary/single star yield ratio for the initial period-secondary mass parameter space for a $7 \mathrm{M}_{\odot}$ primary mass (underabundances only, relative to mass from ISM to stars, $\psi=1$ ).

Stellar Evolutionary Histories

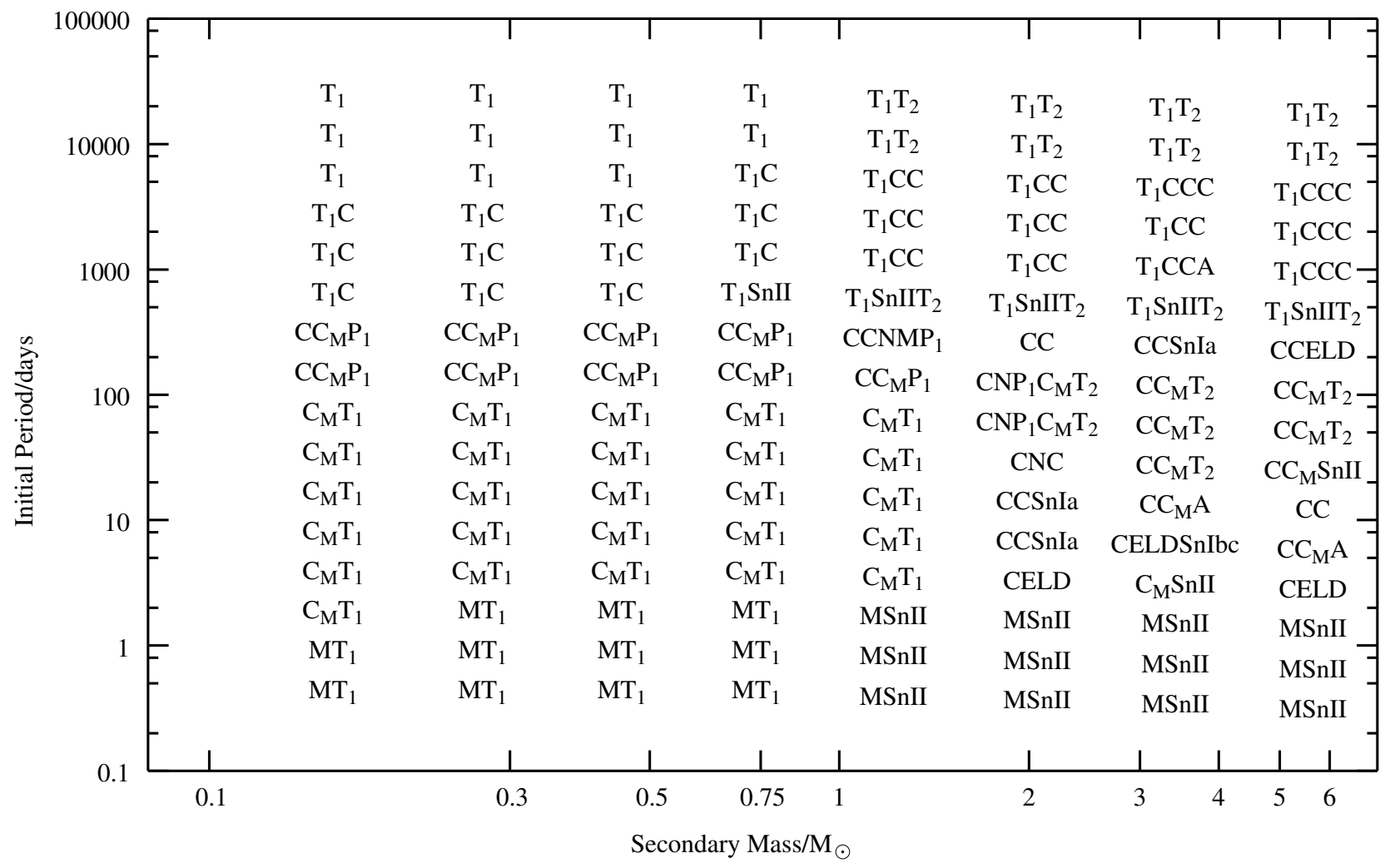

Figure 4 Selected evolutionary histories for stars in Figure 3. T is a TPAGB phase, $C$ common envelope $\left(\mathrm{C}_{M}\right.$ indicates core merger inside the common envelope), $\mathrm{N}$ are novae, Sn are supernovae of type Ib/c, II or Ia when two COWDs coalesce, ELD are possible edge-lit detonations of COWDs by accretion of $\mathrm{He}$, A are accretion-induced collapses, $\mathrm{M}$ any other merger event, $\mathrm{P}$ is a post-common envelope TPAGB star, the subscript 1 represents the primary and 2 the secondary. 
envelope phases; sometimes these lead to new AGB phases. Note that the only systems to significantly overproduce nitrogen are around $M_{2}=1.2 \mathrm{M}_{\odot}, P=350 \mathrm{~d}$ when novae occur causing the nitrogen yield to at most triple; all other systems underproduce or in the case of early mergers (both stars on the main sequence) overproduce by up to $16 \%$. Most systems go through a common envelope phase which causes the truncation of the AGB, some go through two common envelope phases ( $P \approx 250 \mathrm{~d}$ ) which severely limit their yield because their envelopes are thrown off before $\mathrm{CN}$-processing can occur. Companions more massive than about $2 \mathrm{M}_{\odot}$ are more likely to lead to supernovae (types Ia and II) with small nitrogen yields. Initially close binaries merge and if they avoid a supernova contribute roughly the same yields as a single star. Only a few systems $\left(M_{2}>1 \mathrm{M}_{\odot}, P>10^{4} \mathrm{~d}\right)$ have two AGB phases and even these have their yields lowered. The apparent reduction in the ${ }^{14} \mathrm{~N}$ yield at long period is due to the lack of production by the lower mass companion.

\section{Conclusions}

Working with distributions of binary-star parameters typical of what is found in our own Galaxy we have found that the ejecta of binary stars are depleted in ${ }^{14} \mathrm{~N}$ relative to single stars by $36 \%$, with an increase of explosive products ${ }^{13} \mathrm{C}$ and ${ }^{16} \mathrm{O}$ of $70 \%$ and $40 \%$. Switching off the explosions allows us to estimate the effect on AGB stars owing to binary interactions: a drop of $14 \%$ for ${ }^{12} \mathrm{C}, 37 \%$ for ${ }^{13} \mathrm{C}$, and $31 \%$ for ${ }^{14} \mathrm{~N}$. The effect of this reduction is coincidentally cancelled out by supernovae for ${ }^{12} \mathrm{C}$ and swamped by novae for ${ }^{13} \mathrm{C}$. However, the reduction of ${ }^{14} \mathrm{~N}$, the production of which is dominated by AGB stars, is a significant effect which should be included in galactic chemical evolution models. We will shortly be extending the BSE nucleosynthesis package to deal with Wolf-Rayet stars and the s-process in AGB stars.

\section{Acknowledgments}

We thank the referees for a number of improvements to this paper and Gijs Nelemans for useful discussion. RGI is supported by a PPARC grant. CAT is very grateful to Churchill College, Cambridge, for a Fellowship, and the organisers of the Monash-Torino workshop for supporting his attendance. RGI is only slightly jealous.

\section{References}

Bennett, C. L., et al. 2003, ApJ, in press (astro-ph/0302207)

Bondi, H., \& Hoyle, F. 1944, MNRAS, 104, 273

De Donder, E., \& Vanbeveren, D. 2002, NewA, 7, 55

Huang, R. Q., \& Weigert, A. 1982, A\&A, 112, 281

Hurley, J. R., Pols, O. R., \& Tout, C. A. 2000, MNRAS, 315,543

Hurley, J. R., Tout, C. A., \& Pols, O. R. 2002, MNRAS, 329,897

Ivanova, N., Podsiadlowski, P., \& Spruit, H. 2002, MNRAS, 334,819

Iwamoto, K., Brachwitz, F., Nomoto, K., Kishimoto, N., Umeda, H., Hix, W. R., \& Thielemann, F.-K. 1999, ApJS, 125, 439

Izzard, R. G., Tout, C. A., Karakas, A. I., \& Pols, O. R. 2003, MNRAS, submitted

José, J., \& Hernanz, M. 1998, ApJ, 494, 680

Karakas, A. I., Lattanzio, J. C., \& Pols, O. R. 2002, PASA, 19,515

Kroupa, P., Tout, C., \& Gilmore, G. 1993, MNRAS, 262, 545 (KTG93)

Livne, E., \& Arnett, D. 1995, ApJ, 452, 62

Nomoto, K., \& Kondo, Y. 1991, ApJ, 367, L19

Pols, O. R., Schröder, K. P., Hurley, J. R., Tout, C. A., \& Eggleton, P. P. 1998, MNRAS, 298, 525

Portinari, L., Chiosi, C., \& Bressan, A. 1998, A\&A, 334, 505

Vassiliadis, E., \& Wood, P. R. 1993, ApJ, 413, 641

Woosley, S. E., Taam, R. E., \& Weaver, T. A. 1986, ApJ, 301,601

Woosley, S. E., \& Weaver, T. A. 1995, ApJS, 101, 181 